\title{
LA ADMINISTRACIÓN COMO CIENCIA, TÉCNICA Y ARTE
}

\author{
Luis Fernando Valeriano Ortiz (*)
}

E-mail: Ifernandovaleriano@yahoo.es

\section{RESUMEN}

Se viene discutiendo desde hace décadas si la administración es ciencia y arte; ciencia por los conocimientos base que se necesitan para el objeto y estudio de la organización, y arte porque se necesita personalidad y virtudes, aparte de la creatividad. Se suma la técnica porque se necesitan procedimientos y experiencia para la solución de problemas prácticos. Es por esto que la administración se caracteriza por la interdisciplinaridad en todas las carreras y en todos los ámbitos, y en especial en nuestra Facultad.

Palabras clave: Administración, Ciencia, Arte, Técnica, Procedimientos.

\section{ABSTRACT}

It comes discussing since decades ago if the administration was science and art; science by the knowhow base that are needed for the object and study of the organization, and art because is needed personality and virtues aside from the creativity. The technique adds its because procedures are needed and experience for the solution of practical problems. It is for this that the administration is characterized for the interdisciplinary in all the careers and in all the environments, and especially in our Faculty

Key words: Administration, Science, Art, Techniques, Procedures.

(*) Doctor en Ciencias Administrativas. Decano de la Facultad de Ciencias Administrativas de la Universidad Nacional Mayor de San Marcos (UNMSM). 


\section{ANTECEDENTES DEL PENSAMIENTO ADMINISTRATIVO. PRESENCIA DE LA ADMINISTRACIÓN EN LA HISTORIA.}

Si bien es cierto la ciencia administrativa está muy difundida en el escenario actual, y la profesionalización de la ciencia es además reciente, la administración como conocimiento no es una práctica nueva. Desde hace siglos la administración ha sido reflexionada por hombres, imperios, estados-nación y organizaciones, con el propósito de hacer eficiente y eficaz la gestión, además de cumplir objetivos.

En la reflexión histórica de la administración encontramos la necesidad del ser humano de vivir en comunidad para sobrevivir a entornos hostiles; de emprender la búsqueda organizada de recursos y hacerlos productivos; de establecer jerarquías sean tribales, religiosas, militares o políticas y económicas, tal como ocurre hasta nuestros días. Pensemos, sino, en la organización de grupos humanos para la recolección de alimento, la caza y la pesca, actividades con las que el hombre garantizaba su subsistencia y la del conjunto.

Así, junto a la revolución agrícola, el hombre enfrentó la revolución urbana; el influjo de la producción en grupos organizados que posteriormente devinieron en ciudades y estados. Esta construcción social no pudo desprenderse del control, de la planificación, organización ni de la dirección, y sólo pudo ser posible por la presencia de líderes que propusieron objetivos y que establecieron la asignación de recursos.

Tanto estados antiguos como Grecia, Roma, etc., como los modernos organizados en estadosnación como Inglaterra, Francia y España, han tenido clases dirigentes que enfrentaban los problemas de los administradores de hoy: cómo asignar mejor los recursos obtenidos para cumplir o exceder los objetivos propuestos, ejercer autoridad y delegar responsabilidad, además de cumplir los deberes.

\section{ADMINISTRACIÓN CONTEMPORÁNEA}

Es la aparición de la Revolución Industrial la que propone un cambio sustantivo en las técnicas y en la asignación de recursos, haciendo a la producción mucho más eficiente de lo que antes era posible; aparece la reflexión por la productividad, por la eficiencia dirigida y por el control de la producción. Desde finales del siglo XVIII surge la sociedad empresarial, génesis de la empresa corpo- rativa que ahora conocemos y, posteriormente, se crea la necesidad de profesionalizar la gestión.

A principios del siglo XX se establece definitivamente que esta reflexión sobre la organización de la sociedad y de los individuos debe constituirse en objeto de estudio científico. El norteamericano F. W. Taylor (1856-1915), llamado el padre de la administración científica, postula que era la administración y no la fuerza de trabajo el origen y solución potencial a los problemas de la industria. Taylor concluye que los trabajadores usaban siempre tácticas dilatorias porque creían que si trabajaban más rápido se quedarían sin empleo, y porque los salarios por hora o por día destruían el incentivo individual. Pidió una "revolución mental" para fusionar los intereses de los trabajadores con los de la administración en un todo mutuamente beneficioso.

Simultáneamente el europeo H. Fayol desarrolló la llamada teoría clásica de la administración, preocupado por aumentar la eficiencia de su empresa a través de su organización y de la aplicación de una serie de principios generales con bases científicas. Es notable que a pesar de que ambos científicos no hayan tenido contacto entre sí y que hayan partido de puntos de vista diferentes y aún discrepantes, sus ideas constituyan el punto de partida de la denominada Escuela Clásica de la Administración, cuyos postulados dominaron el panorama administrativo de las organizaciones durante las cuatro primeras décadas del siglo pasado, aproximadamente. Posteriormente otras escuelas como la Escuela Burocrática de Weber, la Escuela de las Relaciones Humanas surgida a partir de los estudios de E. Mayo, la Escuela Comportamental o Psicológica de Maslow o la Teoría de Sistemas y de Contingencias, siguen en el camino de profundizar las bases del conocimiento en administración y además validar en un cuerpo único la experiencia de los administradores profesionales.

\section{DIMENSIONES DE LA ADMINISTRACIÓN COMO CIENCIA, TÉCNICA Y ARTE}

El posterior desarrollo de las organizaciones está vinculado con la evolución de la administración, no sólo en su dimensión científica sino en su aplicación y su carácter técnico. Resulta necesario conocer, previamente, el significado de los términos ciencia, técnica y arte para así ponderar el verdadero sitial de la administración en el campo académico. 
- Como ciencia, la administración posee un conjunto de conocimientos organizados sistemáticamente que se basan en la acumulación de conocimiento de larga data y que tiene sus propios principios. Cuenta con un objeto de estudio que es la organización, tiene varios métodos y cuenta con teorías de aplicación general cuyas conclusiones son confiables y susceptibles de adquirir carácter unitario.

- Como técnica, la administración se constituye en un conjunto de procedimientos validados por el conocimiento y por la experiencia, y de aplicación general o particular. Estos procedimientos y recursos que se moldean sobre la base de la profesión de administrador, requieren de un ejercicio constante para adquirir mayor pericia y habilidad al utilizarlos en la solución de problemas prácticos.

- Como arte, la administración se caracteriza por contar con virtud, disposición y personalidad para construir un escenario favorable en una organización de seres humanos con atributos variados y complejos. La administración rescata la necesidad de usar la creatividad en la solución de problemas que impiden el avance de las estrategias y objetivos propuestos. El carácter de arte también la encontramos presente al seleccionar soluciones simples, armónicas e integradoras.

Actualmente podemos decir que la administración es una ciencia porque cuenta con principios que le son característicos, aplicables en cualquier organismo social. Claro está que como disciplina perteneciente a las ciencias sociales tiene que vincularse y apoyarse estrechamente de otros; por lo tanto, el carácter de ciencia, técnica y arte no es excluyente en la administración sino que, por el contrario, deviene en complementario. Conforme la ciencia avanza, también lo hace el arte y la técnica de administrar.

La ciencia administrativa no sólo se alimenta de sí misma; su carácter universal permite recoger experiencias que se unifican en el cuerpo general de conocimientos pero a la vez es flexible en el sentido que procura dar soluciones prácticas a casos particulares. La administración también hace uso de los principios, procesos, procedimientos y métodos de otras ciencias que están relacionadas con la eficiencia en el trabajo. Adicionalmente está relacionada con matemáticas, estadística, derecho, economía, contabilidad, mercadeo, sociología, psicología, filosofía, antropología, etc.
Esta multidisciplinaridad e interdisciplinaridad nos señalan estrategias de cooperación entre dos o más disciplinas en la resolución de un proyecto o problema de investigación, así como explican (especialmente la interdisciplinaridad) los nuevos temas o problemas que se forman en la intersección que ocurre en la periferia entre dos o más disciplinas, pero no constituyen por sí mismas transformaciones epistemológicas que conmocionen el mapa científico.

\section{LA ADMINISTRACIÓN EN LA UNIVERSIDAD NACIONAL MAYOR DE SAN MARCOS}

La Facultad de Ciencias Administrativas de la Universidad Nacional Mayor de San Marcos (UNMSM) es conciente del rol que tiene en la construcción de la ciencia y en el desarrollo de la profesión; por ello, nuestra Facultad responde a la formación de profesionales licenciados en Administración, Administración de Negocios Internacionales y Administración de Turismo.

La Licenciatura en Ciencias Administrativas está diseñada y orientada hacia el desarrollo de profesionales con conocimientos integrales actualizados y con liderazgo emprendedor basado en el fortalecimiento de valores éticos y en la continua construcción de habilidades y competencias que le permitan una comprensión gerencial sistemática de los procesos y problemas, así como una visión estratégica para abordar los complejos temas de decisiones y soluciones a nivel personal, profesional, empresarial y cívico.

La contribución de nuestros docentes apunta principalmente a la comprensión de los problemas de las organizaciones, sean de carácter público o privado, sean grandes corporaciones o pequeñas empresas pero, además de la comprensión, nuestros docentes aportan su experiencia práctica en la solución de los problemas analizados desde el punto de vista del gestor, del administrador o del empresario, donde prima el cumplimiento de los objetivos trazados, la eficiencia, la eficacia y la sostenibilidad en entornos complejos y cambiantes como los de hoy.

La formación en ciencias administrativas de nuestra Facultad, como en toda carrera, considera la participación de profesionales de otras ciencias porque la multidisciplinaridad es una estrategia de cooperación y no una disolución de las ciencias administrativas que puede garantizar una alta especialización en la formación de admi- 
nistradores y gerentes, integrando los aportes de otras ciencias para la solución de los problemas prácticos y creando nuevos enfoques en el desarrollo científico de la administración frente a los desafíos del mundo global. 